\title{
Flow Cytometry and DNA Study: A Powerful Tool in Medical Conditions
}

\section{Vasiliki E Kalodimou*}

Head of Flow Cytometry-Research and Regenerative Medicine Department, laso Maternity Hospital, Athens, Greece

Flow cytometer system analyze biological specimen in order to identify various cell populations determined by the specific monoclonal antibodies and fluorochromes used. A flow cytometer is made up of three main systems: fluidics, transports particles in a stream to the laser beam for interrogation, optics, consists of lasers to illuminate the particles in the sample stream and optical filters to direct the resulting light signals to the appropriate detectors, and electronics, converts the detected light signals into electronic signals that can be processed by the computer [1-3].

In the flow cytometer, particles are carried to the laser intercept in a fluid stream. Any suspended particle of cell from 0.2-150 micrometers in size is suitable for analysis. The characteristics or parameters of each event are based on its light scattering and fluorescent properties $[3,4]$. The data are collected and stored in the computer. This data can be analyzed to provide further information about subpopulations within the sample.

The analysis of DNA, after self-fluorescence, is the most important measurement in flow cytometry. Changes in DNA often accompany many malignant diseases of the blood (e.g., acute leukemia, myelodysplastic syndromes, lymphoma, multiple myelomas, etc), and there are studies investigating the possible prognostic value of ploidy in human tumors, (e.g., cardiac myxoma, colon tumors, breast tumors, stomach tumors, urinary tract tumors, etc), that are formed inside the cell cycle.

Flow cytometry measures the percentage of cells found in each phase of the cycle (analysis of the cell cycle) and the total DNA content of the cell population (cell content of DNA or DNA ploidy). Linking DNA and dye is stoichiometric; that is, the amount of dye that is used is proportional to the amount of DNA in the cell. The fluorescence intensity is proportional to the amount of dye that is bound to DNA.

The reaction of DNA to dye is bidirectional, so the measurement must be performed at constant temperature conditions on the cytometer. To measure the amount of DNA, cells must be labeled with a fluorochrome that has the capacity to bind to DNA in a way that gives us the absolute number of its quantity.

After staining, the samples can be measured in the cytometer, using appropriate settings and protocols, in order to generate the DNA histogram. The highest peak refers to the cells at the G0/G1 phase, the second highest is for the gap 2/mitosis (G2/M) phase cells, and the region between them is for the cells located in the synthesis (S) phase. Additional peaks reveal the existence of aneuploidy.

The $S$ phase is particularly important because it reflects the proliferative activity of tumors. The results of the analysis are mainly expressed by the percentage of cells in the $S$ phase compared to the total number of cells and the proliferation index, which indicates the percentage of cells in the $S$ and G2/M phases.

Normal somatic cells contain a diploid chromosome number (2n). The neoplastic cells are characterized by genetic variability. An abnormal number of chromosomes is referred to as aneuploidy and reflects changes in the DNA content of a cell. Hyperdiploid refers to cells with a chromosome number $>2 \mathrm{n}$, and hypodiploid refers to cells with chromosome number $<2 \mathrm{n}[5]$.

The fluorochrome most commonly used for DNA staining is Propidium Iodide (PI), which has red fluorescence and is emitted up to $488 \mathrm{~nm}$. PI has two disadvantages: 1) it double stains all nucleic acids, so the cells have to be incubated with RNase, and 2) it also cannot label the cell membrane, causing the cells to be permeable before its addition, for example, with $70 \%$ ethanol.

We can also use 4', 6-diamidino-2-phenylindole (DAPI), which results in high-quality histograms, for DNA staining; this process requires ultraviolet (UV) fluorescence or a violet-colored laser. Recall that for the proper use of fluorochromes and to get high-quality DNA histograms, we must know the right length of incubation and the correct concentration of the cells we want to use.

The concept of flow cytometry uses DNA aneuploidy as a result of the change in the amount of DNA and, of course, the changes in chromosome number. An important characteristic is that disturbances of the ploidy can be detected by flow cytometry only when the amount of DNA exceeds a certain threshold, with at least three chromosomes (from 46 to 43 or 49 chromosomes). A DNA index equal to the unity may mean a normal, slightly disturbed, or very irregular karyotype. Flow cytometry cannot show the structural changes of chromosomes. The content of DNA in a tumor should be expressed as the DNA index, which is defined as the ratio of the DNA in a cancer cell compared to a normal diploid cell [5].

The quality of DNA histograms is estimated from the peak of the width of the DNA in cells in the G1 phase of the cell cycle. This measurement is based on the coefficient of variation $(\mathrm{CV})$ and is calculated from the standard deviation $(\mathrm{SD}): \mathrm{CV}=100 \times \mathrm{SD} /$ (peak channel)\%, where the top of the peak of the channel is the average of the peaks. The smaller the number of peaks in the CV in a DNA histogram is, the more likely it is that the measurements of ploidy are absolutely correct and the more likely it is that we can calculate the absolute cell populations in each phase of the cell cycle. To avoid false results, the flow cytometer must be properly aligned, and any debris in the sample must also be eliminated. The keys to achieving the best quality histogram are proper sample preparation, using the correct setting for the machine, and correct interpretation of the results.

The quality of the results is directly correlated to proper operation of the machinery; therefore, the flow cytometer parameters should be monitored daily using fluorescent beads and a known CV. The

*Corresponding author: Kalodimou VE, Head of Flow Cytometry-Research and Regenerative Medicine Department, IASO-Maternity and Research Hospital, Kifisias Avenue 37-39, 151 25, Marousi, Athens, Greece, Tel: 0030-210-6185203; Fax: 0030-210-610-5711;E-mail: kalodimou@yahoo.gr

Received August 28, 2013; Accepted August 29, 2013; Published September 04, 2013

Citation: Kalodimou VE (2013) Flow Cytometry and DNA Study: A Powerful Tool in Medical Conditions. Cardiol Pharmacol 2: e115. doi:10.4172/2329-6607.1000e115

Copyright: (C) 2013 Kalodimou VE. This is an open-access article distributed under the terms of the Creative Commons Attribution License, which permits unrestricted use, distribution, and reproduction in any medium, provided the original author and source are credited. 
quality of the DNA histogram is estimated by the CV for the G0/G1 division. Achieving a perfect $\mathrm{CV}$ is crucial to demonstrate the existence of discernible peaks in the distribution of DNA aneuploidy in tumor cells compared to normal DNA diploid cells, which coexist in varying proportions. Excluding double cells is also essential because they are recognized by the cytometer as a cell with increased DNA. For this reason, measurements are set at a low flow rate. Any disruption of the sample to be analyzed by flow cytometry increases the CV, resulting in a high cell concentration, between $5 \times 105$ and $2 \times 106 / \mathrm{mL}$, while the flow rate remains low. The DNA histogram must be presented only on a linear scale [5].

In conclusion DNA analysis by flow cytometry provides fast results, permits multiparameter analysis correlating DNA content with antigen expression, and provides the sensitivity for detecting neardiploid aneuploid peaks.

\section{References}

1. Ormerod MG (2000) Flow cytometry: A practical approach. 3rd edition, Oxford University Press.

2. Vindelov LL, Christensen IJ, Nissen NI (1983) A detergent-trypsin method for the preparation of nuclei for flow cytometric DNA analysis. Cytometry 3: 323327.

3. Rabinovitch PS (1994) DNA Analysis: Darzynkiewicz Z, Robinson JP, Crissman HA, editions. Flow cytometry. Methods in cell biology, Volume 41, Academic Press, San Diego, USA.

4. Ormerod MG, Titley JC, Imrie PR (1995) Use of light scatter when recording a DNA histogram from paraffin-embedded tissue. Cytometry 21: 294-299.

5. Kalodimou VE (2013) Basic Principles in Flow Cytomety. American Association of Blood Banks, USA. 